\title{
X-ray free-electron lasers for the structure and dynamics of macromolecules
}

\section{Henry N. Chapman,,$^{1,2,3}$}

${ }^{1}$ Center for Free-Electron Laser Science, DESY, Notkestrasse 85, 22607 Hamburg, German; email: henry.chapman@desy.de

${ }^{2}$ Department of Physics, University of Hamburg, Luruper Chaussee 149, Hamburg, 22761, Germany

${ }^{3}$ Centre for Ultrafast Imaging, University of Hamburg, Luruper Chaussee 149, Hamburg, 22761, Germany

Xxxx. Xxx. Xxx. Xxx. YYYY. AA:1-25

https://doi.org/10.1146/((please add article doi))

Copyright (c) YYYY by Annual Reviews. All rights reserved

\section{Keywords}

structure determination, time-resolved crystallography, serial crystallography, coherent diffraction, radiation damage, FEL

\section{Abstract}

X-ray free-electron lasers provide femtosecond-duration pulses of hard X-rays with a peak brightness about one billion times greater than available at synchrotron radiation facilities. One motivation for the development of such sources was the proposal to obtain structures of macromolecules, macromolecular complexes, and virus particles without the need for crystallisation, through diffraction measurements of single non-crystalline objects. Initial explorations of this idea and outrunning radiation damage with femtosecond pulses led to the development of serial crystallography and the ability to obtain high-resolution structures of small crystals without the need for cryogenic cooling. Using the technique, conformational dynamics, enzymatics, and the resolution of intermediate states in reactions can be accomplished over timescales of $100 \mathrm{fs}$ to minutes. Challenges remain to do the same without crystals, with the promise of more diffraction photons recorded per atom than electrons contributing to an molecular image. 


\section{Contents}

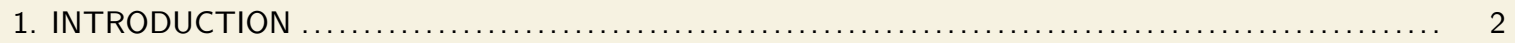

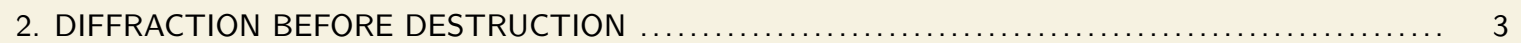

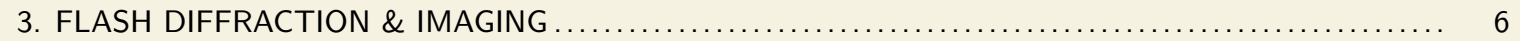

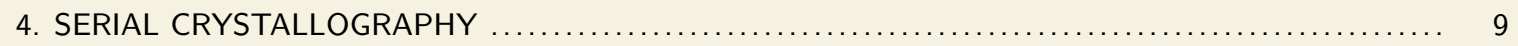

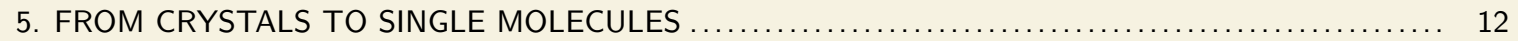

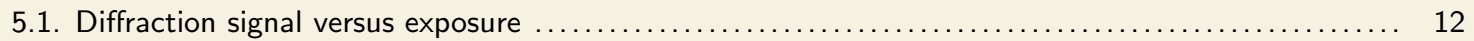

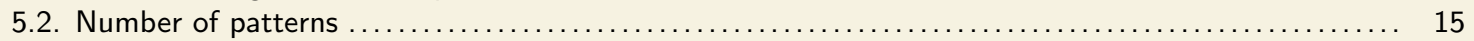

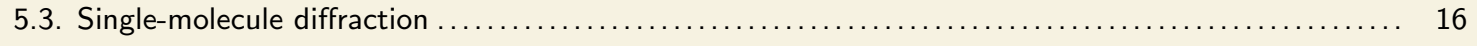

\section{INTRODUCTION}

The methodologies to gain three-dimensional images of molecules with atomic detail were among the towering scientific achievements of the twentieth century. This began with the discovery of X-ray diffraction from crystals, leading to the acceptance of X-rays as short-wavelength electromagnetic waves and the realisation that the arrangements of atoms in a crystals could be deduced from such measurements. With the crystal structures of hemoglobin and myoglobin obtained with great effort in the 1950's, many other breakthroughs followed to establish macromolecular crystallography as the present dominant approach to structure determination (1). In early 2018 the protein databank (PDB) (2) listed over 140000 depositions of structures, of which more than 125000 were determined using X-rays.

Crystallography fortuitously solved two problems that would have otherwise prevented atomic-resolution structures to be obtained. Firstly, it avoided the need for an atomicresolution lens to form images of molecules, although in doing so created the so-called "phase problem" of how to synthesise those images from the diffraction measurements (see IMAGING WITHOUT LENSES). Perhaps less obviously, crystallography allowed structural information to be obtained at the atomic scale without excessively suffering from the effects of bombarding the molecules with ionising radiation. The short wavelengths that are required to be able to resolve two atoms separated by the length of their bond means that $\mathrm{X}$-ray photons have energies that significantly exceed the thresholds to liberate core electrons from the lighter elements that make up organic matter, and hundreds of times greater than that needed to break bonds. Such photon energy absorption therefore wreaks havoc to the very structure under scrutiny, and in fact happens much more frequently than elastic scattering from atoms, the events which contribute to the diffraction patterns used for structural analysis. By arraying billions of identical molecules into a crystal, the molecular scattering pattern is amplified in proportion to their number, whereas the average number of bonds broken per molecule can be kept almost arbitrarily small during the exposure (for an arbitrarily large crystal). Of course, given the low strength of the X-ray tubes that existed in the early days of crystallography, even long exposures usually did not cause much damage to the sample. Large crystals were needed because the X-ray sources were feeble.

As X-ray technologies progressed in the familiar exponential trend of these times, with electron storage ring facilities generating beams of ever greater brightness, protein crystal sizes could be reduced to volumes of hundreds of cubic micrometers and the effects of radiation damage became more apparent even with short exposures (3). Cryogenic temperatures 
increased the dose that a biological specimen could tolerate by about a thousand fold before its structure was affected at high resolution $(4,3)$. Concurrently, cryogenic protection enabled the imaging of uncrystallised macromolecules by transmission electron microscopy (TEM), where the cooling also immobilised samples (embedded in vitreous ice) over the duration of the exposure (5). TEM lenses could certainly form images with atomic resolution, avoiding the phase problem. As with X-rays, the short de Broglie wavelength corresponds to energetic ionising particles, but the interaction of electrons with matter are much more favorable. The amount of energy deposited in the sample, and hence the degree of damage, for a given elastic scattering contribution to an image is vastly reduced as compared with $\mathrm{X}$-rays. This is enough to obtain measurable images from single molecules, albeit with signal levels that are barely distinguishable from noise. A key insight was that the requirement for the image strength was only that the positions and orientations of molecules could be extracted from the images so that many could be combined (to form a three dimensional image) and noise averaged away (6). Recent technological progress, known as the "resolution revolution," provides a more assured way to gain structural insights for samples that cannot be readily crystallised, and has become the preferred approach for examining the structures of many protein complexes (7).

With the development of X-ray free-electron lasers (FELs) (8) (see "X-RAYS FROM LINEAR ACCELERATORS"), which produce brief and intense flashes of X-rays, another strategy came to bear for avoiding the effects of radiation damage, with the potential to match signal levels achievable in cryo-electron microscopy. Instead of using low temperature to keep the structure intact, the principle is to use short times to "freeze" X-ray induced motion of atoms in the sample. The time needed to achieve this depends on how fast atoms move, which in turn depends on their inertia (their mass) and the forces applied to them. If the exposure is so high as to ionise every single atom, then the Coulombic forces dictate speeds approaching $0.1 \AA \mathrm{fs}^{-1}$ of the lighter elements, requiring femtosecond pulses, which can indeed be generated by FELs. By being able to perform measurements on warm samples, at essentially any useful temperature, it becomes possible to follow the evolution of structures undergoing reactions and to determine the influence of temperature and environment on the conformations that a system may adopt. Much of this new field is yet to be developed, and the extremes of the technique have not yet been reached. However, it is useful to recount many of the formative steps as well as to consider the use of X-ray FEL radiation in juxtaposition with crystallography and cryo-electron microscopy.

\section{DIFFRACTION BEFORE DESTRUCTION}

An early suggestion of using short intense X-ray pulses for examining biological structures was put forth in 1975 by Wood and Chapline $(9,10)$, at the dawn of research into Xray lasers (including X-ray FELs). The high spatial coherence of such laser sources was seen as a great benefit as it would allow holographic imaging of biological samples to overcome the phase problem of diffraction measurements, as well as offering pulses of about 1 fs duration $\left(10^{-15} \mathrm{~s}\right)$ that could freeze molecular vibrations (10). The authors were perhaps unaware of an earlier analysis by Breedlove and Trammell (11) who concluded that femtosecond-duration exposures would be required for the imaging of single macromolecules with electrons, but would offer no advantage for X-rays due to the large number of ionising events that take place for every scattered photon, no matter what the exposure time. Such concerns were countered by the realisation that the inertia of atoms actually gives some

www.annualreviews.org $\bullet X$-ray FELs 
time before blurring and sample destruction becomes apparent $(12,13,14)$, at least at the nanometer scale. By the time that the construction of X-ray FELs was being considered in the 1990s, these considerations suggested that the femtosecond-duration pulses of FELs could allow atomic resolution, following from a simple dependency of imaging resolution on exposure time (15). Doniach (16) carried out such an extrapolation to atomic scales to conclude, like Breedlove and Trammell, that the excess of ionisations over scattering events would unfortunately invalidate such hopes for imaging of molecules, unless they were arranged in a small crystal. Thus, the overall interest in the potential of FELs for structural biology, discussed in a July 1999 meeting at DESY, Hamburg, was somewhat tempered by caution (17). The meeting may have stimulated new thoughts, however, since by Christmas that year Janos Hajdu and colleagues submitted a paper (18) proposing a scheme to achieve structure determination from a series of FEL diffraction measurements of single molecules. Molecular dynamics simulations showed that in such small samples the photoelectrons initially escape the molecule (further reducing the damage occurring over the duration of the pulse). The objection of Breedlove, Trammell, and Doniach was overturned by adopting the strategy of single-molecule cryo-electron microscopy to average much weaker patterns than they had thought necessary (here in diffraction space instead of the real space of electron microscopy), obtained from many individual macromolecules in a serial fashion ${ }^{1}$. The paper and subsequent work by Hajdu and his collaborators contributed to a strong impetus for the construction of the Linac Coherent Light Source (19), a project that was approved by the US Department of Energy in 2000, as well as the SACLA (20) and the European XFEL (21). These facilities opened for users in 2009, 2012, and 2017, respectively.

The peak intensity of X-ray beams that were to be produced by FELs was unprecedented and posed immediate questions of the response of matter and even if instrumentation would survive more than a single pulse. Experimenters were used to the conditions at synchrotrons

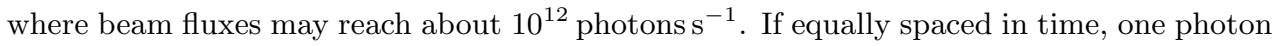
would arrive on the sample per picosecond, separated by the $300 \mu \mathrm{m}$ distance light travels in that time. If focused to a spot of $10 \mu \mathrm{m}$ diameter, the beam would contain one photon per $30000 \mathrm{~mm}^{3}$. For a protein crystallography experiment on a small crystal, at any instant of time the sample is therefore likely not interacting with an X-ray photon, and only occasionally a lone photon passes through the sample. The situation is quite different with an X-ray FEL pulse, which can be focused to a diameter of $0.1 \mu \mathrm{m}$. All $10^{12}$ photons (or more) may arrive in an FEL pulse of $30 \mathrm{fs}$ duration, giving a single photon in each $0.01 \mathrm{~nm}^{3}$ volume of the beam. That is, the photon density is about equal to the density of atoms in condensed matter. As soon as one photon passes by an atom, another impinges, and this is true for all atoms in the sample.

$\mathrm{X}$-rays must interact with atoms to be able to discern any information about the structure of an object (such as through X-ray diffraction). X-rays interact in several ways, and at wavelengths of about $1.5 \AA$ ( $8 \mathrm{keV}$ photon energy) the most likely response is for an atom to absorb the photon, resulting in the ejection from the atom of an electron of slightly lower energy than the photon. The process is incoherent, and primarily adds a featureless background to a diffraction measurement ${ }^{2}$. The probability of this occurrence is expressed as a cross section, which indicates how big a target the atom appears to be. The photoab-

\footnotetext{
${ }^{1}$ By averaging noisy but oriented images, cryo-electron microscopy avoided the short pulses that Breedlove and Trammel deemed necessary.

${ }^{2}$ But see Classen et al. (22) for a proposal to extract structural information from fluorescence.
} 
sorption cross section for an oxygen atom for $8 \mathrm{keV}$ photon energy is $\sigma=3.0 \times 10^{-14} \mu^{2}$ which is to say that with a beam of $3.3 \times 10^{13}$ photons $\mu \mathrm{m}^{-2}$ an atom will likely absorb one of those photons. This is true for any atom exposed to the beam, meaning that all atoms in an illuminated object are likely to absorb a photon. The ionised atoms, missing a core electron, relax by rearranging their electronic configurations to the ground state, resulting in further emission of a fluorescence photon or an Auger electron. The time for this relaxation to take place is several femtoseconds, before which the atom is less likely to absorb another photon(23). It is thus natural to consider a saturation intensity, whereby every atom is simultaneously empty of a core electron, equal to $E_{\nu} /(\sigma \tau)$, where $\tau$ is the lifetime of the core hole state, and $E_{\nu}$ is the photon energy. In oxygen $\tau$ is approximately $3 \mathrm{fs}$, giving a saturation intensity of $1.1 \times 10^{13}$ photons $\mathrm{mm}^{-2} \mathrm{fs}^{-1}$. In other units this is a power density of $1.4 \times 10^{21} \mathrm{~W} \mathrm{~cm}^{-2}$ (for $8 \mathrm{keV}$ photons), achievable by focusing a typical XFEL pulse to a spot size of about $0.1 \mu \mathrm{m}$.

Although absorption is the most common process, this only delivers energy into the sample. Structure is encoded in the interference of X-rays that elastically scatter from the atoms in a sample (the diffraction pattern). This scattering process is different and less probable than photoabsorption, occurring only once for every 32 atoms undergoing photoabsoption for our example of oxygen atoms irradiated with $8 \mathrm{keV}$ photons. For every $8 \mathrm{keV}$ photon that contributes to a diffraction pattern, there are 32 photons worth $(256 \mathrm{keV})$ of energy absorbed in the sample. A recording of at least one scattered photon per atom would therefore require $256 \mathrm{keV}$ /atom to be absorbed. This intrinsic quantity is known as dose, equal to 48 GGy in SI units. It is the dose, energy deposited per unit mass, that is ever linked to the strength of the diffraction signal as Breedlove and Trammel pointed out, and which causes a particular degree of sample degradation. A diffraction measurement is much like reading a message on light-sensitive paper, fading in the light needed to see it. The solution provided by FELs is to read the message in a flash. Photoabsorption still occurs, even with femtosecond pulses, but as long as the atoms remain in place and can scatter X-rays, this may not effect the pattern.

There are still limits. The saturation intensity, photoionising every atom within their fluorescence lifetimes, would provide about 0.03 diffracted photons in the pattern per atom. Even in this case there are still electrons bound to atoms which can continue to interact further with X-rays, and the ultimate limit would be when there are no more electrons left in the sample to scatter from. The scattering cross section decreases as electrons are removed, requiring even higher X-ray intensities to achieve stronger diffraction signals. Son et al (23)

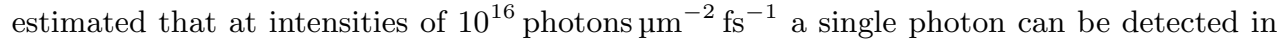
a diffraction pattern per carbon atom in the sample, and that additional diffraction signal would develop in proportion to $I_{0}^{0.57}$, where $I_{0}$ is the pulse intensity equal to the number of incident photons per unit area and unit time. As yet, no FEL experiment has reached these high intensities.

When exposures are longer than several femtoseconds, the electrons emitted from the ionised atoms become the dominant destructive forces acting upon other atoms in the sample. A photoelectron from a light element is energetic enough to inelastically scatter from a neighbouring atom, ejecting a valence electron from this atom, setting off a cascade of ionisations that take place over a distance of about $1 \mu \mathrm{m}$ and taking about $100 \mathrm{fs}$ to complete $(24,25)$. The consequence of each photoabsorption event can be up to 200 ionised atoms, causing disruption through the breaking of bonds and motion of atoms as the entire sample transitions into a plasma state. For such "long" exposures of tens of femtoseconds, 
a safe exposure may therefore be at the level at which all atoms are ionised (mainly from electron collisions) by the end of the pulse. During the exposure, X-ray scattering will thereby mostly occur from pristine atoms. For a typical protein, this corresponds to a dose of about $400 \mathrm{MGy}$ (26). FEL experiments in this intensity regime have revealed specific changes near heavy elements $(27,28)$, as discussed below.

Based only upon arguments considering the interaction of X-rays with atoms, it is seen that both the strength of diffraction and the degree of X-ray induced structural changes depend on the incident pulse fluence (number of photons per unit area) but by squeezing this fluence into a shorter pulse always reduces the degree of degradation that occurs over the course of the exposure. That is, higher intensity is always better.

\section{FLASH DIFFRACTION \& IMAGING}

The first opportunities to explore Wood and Chapline's ideas of single-shot or "flash" X-ray imaging of biological materials arose in the early 1990's with soft X-ray lasers pumped by large optical lasers. A facility at Princeton University, producing pulses of soft X-rays with a similar number of photons as an XFEL of today but of a million times longer duration, was used to make contact images of objects in photoresist (29). At the Nova laser of Lawrence Livermore National Laboratory an image was formed with a shorter 200 ps exposure of a gold-labelled sperm cell using a zone plate diffractive lens (30). The X-ray laser beam was more dilute, by nine orders of magnitude, than the "atomic" photon density of focused XFEL pulses and no visible damage of the samples was reported. The investigation of radiation damage at much higher exposure levels (and doses) had to wait until 2005, when the first soft X-ray FEL, at the Tesla Test Facility at DESY in Hamburg, Germany, opened as a user facility (31). Later renamed FLASH, this facility could generate thousands of high-intensity femtosecond-duration soft X-ray pulses per second rather than one Nova pulse every few hours (32).

Although the X-ray wavelengths at FLASH were too long to access structural information at the molecular scale, the motivation for the first imaging experiments at that facility was to test the feasibility of Hajdu and colleagues' serial diffraction scheme (18) and to spur development of the necessary technologies in anticipation of upcoming shortwavelength FELs. Pressing questions were the possibility to capture weak but interpretable diffraction signals in the face of pulses that would vaporise everything in their path (possibly including the instrumentation and sensitive detector), and whether indeed the exposure time would be faster than the relevant damage processes. Another goal was to demonstrate "lensless" imaging of general non-crystalline objects from their far-field continuous diffraction patterns, utilising the improved spatial coherence of FEL sources (see Figure 1).

A collaboration between the groups of Hajdu and Chapman demonstrated these goals soon after FLASH was opened as a user facility. They recorded a coherent diffraction pattern of a test object - a design etched into a $20 \mathrm{~nm}$ thick silicon nitride membrane - with a single $25 \mathrm{fs}$ duration pulse of $32 \mathrm{~nm}$ wavelength, focused to a power density of $4 \times 10^{14} \mathrm{~W} \mathrm{~cm}^{-2}(38)$ - The pattern was easily phased directly to reveal an image of $62 \mathrm{~nm}$ resolution that well matched a previously-recorded electron micrograph. The object was destroyed in the process of imaging; the diffraction pattern from a second pulse revealed a large hole where the object had been. Damage to the instrumentation itself was avoided with a novel camera system based on a multilayer-coated mirror with a hole that allowed the intense beam to pass harmlessly through the experiment (39). While gratifying, it was not surprising to out-run

6 Chapman 


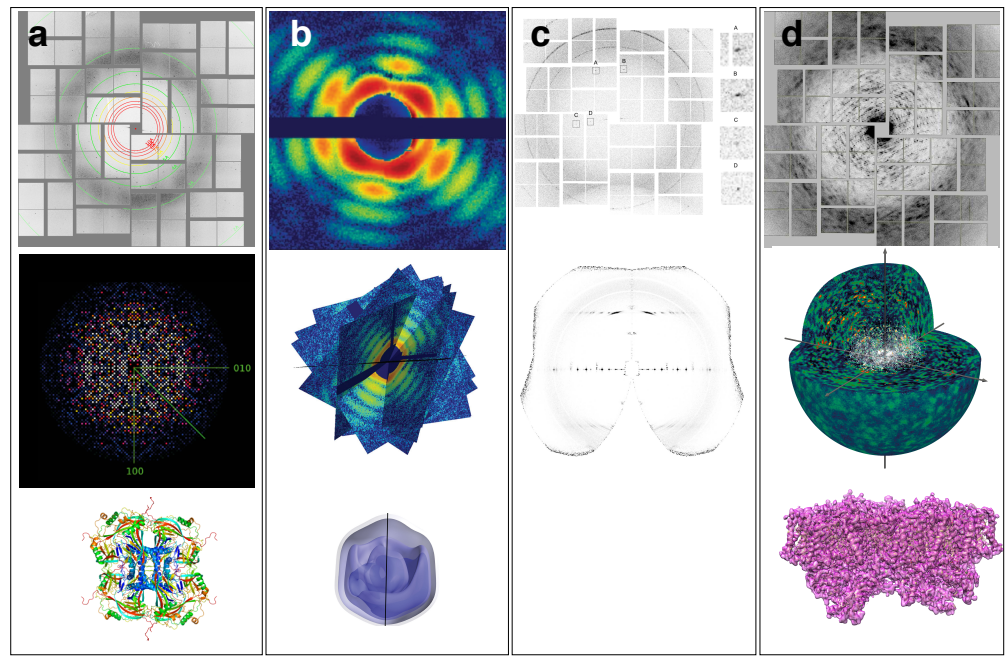

Figure 1

Examples of X-ray FEL diffraction: (a) granulovirus crystals (33), (b) single mimi virus particles $(34,35)$, (c) crystalline fibres (36), and (d) translationally disordered photosystem II crystals (37). Single-shot patterns are shown in the top row, aggregated data assembled 3D reciprocal space in the middle, and the structure determined from each of these along the bottom, except in (c).

destruction at a length scale of $60 \mathrm{~nm}$ with a $25 \mathrm{fs}$ pulse. A museum exhibit of Newton's dusty mirror experiment provided inspiration to improve the test to $3 \mathrm{~nm}$ precision (40), showing particle expansions below $6 \mathrm{~nm}$ in $350 \mathrm{fs}$, at an X-ray dose of about $500 \mathrm{MGy}$, in agreement with hydrodynamic models.

An aerodynamic lens stack developed for aerosol mass spectrometry at Lawrence Livermore National Lab was adopted for low-background particle diffraction experiments at FLASH (41). A simple aperture in a plate constricts the flow of gas (and entrained particles), tending to move high-momentum particles onto streamlines close to the aperture axis. The lens stack, consisting of several thin-plate apertures aligned to the central axis of a long narrow tube, produced particle beams of about $20 \mu \mathrm{m}$ diameter and speeds up to $150 \mathrm{~m} \mathrm{~s}^{-1}$. Diffraction patterns are recorded by chance when a particle intersects the beam focus in coincidence with an XFEL pulse, which for the initial FLASH experiments only occurred once every two minutes.

Despite its long wavelength, which was eventually reduced down to $4.2 \mathrm{~nm}$ at the $K$ shell absorption edge of carbon, FLASH proved to be an effective platform for developing the methodologies for single particle diffractive imaging and exploring new ideas for imaging (42). A limitation for these developments, however, was that the large objects observable at low spatial resolution are not generally reproducible at these length scales. Only very simple objects, such as ellipsoidal iron oxide particles, helped develop an algorithm to register diffraction patterns from objects in random and unknown orientations so they could be summed into a three-dimensional set of structure factors (43).

As the 2009 opening of the Linac Coherent Light Source (LCLS), the world's first hard $\mathrm{X}$-ray FEL, drew near, one pressing question still remained: does the concept of "diffraction 
before destruction" apply to atomic length scales? The experiences at FLASH saved several years of development and stimulated the preparation of a direct-dection pnCCD system for diffraction measurements at high frame rate. Built by the Max Planck Society, an instrument containing two such detector planes in vacuum was made available by Joachim Ulrich and Ilme Schlichting to the Atomic and Molecular Optics (AMO) beamline of LCLS where the first beam would be delivered (44). Although designed in part to continue the research program of single particle imaging with the more energetic and shorter-wavelength pulses of LCLS, we proposed that structural integrity could be better tested at atomic scales quite simply by monitoring the diffraction from macromolecular crystals. Bragg peaks depend upon the constructive interference of waves scattering from the many repeating units in a crystal, and will only occur at a particular scattering angle if those units are reproducible. Destruction of the sample would destroy crystalline order, starting at the finest length scales as observable by loss of Bragg peaks at highest scattering angles and progressing to lower angles, which could be read off the diffraction pattern. This proposal was made possible by a parallel effort led by John Spence of Arizona State University to accumulate electron or X-ray diffraction of single molecules in solution, but aligned to the laboratory frame by the action of an intense polarised laser beam $(45,46)$. A cumulative exposure over this solution of molecules would give rise to diffraction scaled up from that of a single molecule, even with significant background scattering that would arise from the liquid. As in crystallography, rotating the laser polarisation to rotate the molecules gives three-dimensional structural information. By the time of the LCLS opening, the ASU team had adopted a design of a coaxial sheath of gas that focused the liquid into a jet much thinner than the nozzle opening $(47,48)$ to reduce the background scattering. The beauty of this system was that micro-crystals could be provided to the X-ray beam in a liquid medium (the crystallisation liquor) at room temperature but into a vacuum environment. A cleverly designed tube, enclosing the nozzle and liquid jet, replaced the aerodynamic lens stack in the LCLS experiment and provided a long flight tunnel for the spent liquid to freeze out on a cryogenic pump. The first attempt to measure diffraction at LCLS took place in December, 2009. The experiment worked flawlessly to provide over three million recorded diffraction frames from the the first sample tried-photosystem I nano-crystals produced by Petra Fromme.

Measurements immediately showed Bragg peaks to the edge of the detector, proving that structural information could indeed be recorded at a dose of about $700 \mathrm{MGy}$, about 1000 times greater than what a room-temperature crystal could usually tolerate. At that time the LCLS provided photon energies only up to about $2 \mathrm{keV}$ ( $6 \AA$ wavelength) so the test was limited to a resolution of $8.5 \AA$ (49). It was further found that lengthening the X-ray pulses beyond $100 \mathrm{fs}$ reduced the strength of high-resolution Bragg peaks but never completely extinguished them. This presented a puzzle: how could structural information survive such long pulses? The answer was that during the initial moments of the pulse, prior to the onset of disorder, constructive interference to create Bragg peaks could occur (50). The destruction thus gates the measurement - the latter parts of the pulse do not influence the Bragg intensities, but a complete turn off can only happen if the disorder progresses isotropically. The second sample tried in the same week of LCLS beamtime, lysozyme nano-crystals produced by Ilme Schlichting, showed a more complicated response (51), and measurements made at shorter wavelengths showed that specific atoms, such as those surrounding a heavy atom (which is thus strongly ionising) can move along preferred trajectories (27), in agreement with calculations (52). Due to their high inertia, heavy atoms in a protein may stay 
in place even as the structure of lighter elements melts around them. The heavy atoms, still forming a lattice, would thus continue to scatter into Bragg peaks albeit with modified structure factors which add incoherently to those of the pristine structure. Such counterintuitive effects may even be amplified by the continuous neutralisation of heavy atoms which draw electrons from surrounding atoms (28) to "recharge" their scattering strength. The lesson learned was that measurements must be made with pulses shorter than about $20 \mathrm{fs}$, especially for molecules containing heavy elements, to avoid systematic errors in atomic coordinates.

The second week of the extraordinary December 2009 run at LCLS was devoted to the original scope of imaging aerosolised non-crystalline particles such as mimi viruses, using an aerodynamic lens built at Uppsala University to stream them across the XFEL beam (53). The iterative phasing algorithms honed at FLASH revealed snapshot images of single mimi virus particles, $450 \mathrm{~nm}$ in diameter, to a resolution of $32 \mathrm{~nm}$. Steady development of the method has since been continued at LCLS and SACLA, greatly helped with the Single Particle Initiative - a community effort carried out under the auspices of the LCLS facility $(54,55)$ - as discussed further in Sec. 5.

\section{SERIAL CRYSTALLOGRAPHY}

The first diffraction experiments carried out at LCLS in 2009 brought forth a new and unanticipated methodology for structural biology, which formed the basis for many further developments and studies at all hard X-ray FEL facilities (56). However, it was not until this approach was demonstrated at the higher photon energies of about $8 \mathrm{keV}$ available at the CXI beamline at LCLS in 2010 (57) that the hesitation voiced at the 1999 DESY meeting could finally be assuaged. The refinement of the structure of lysozyme from the diffraction patterns of 12000 individual nano-crystals, recorded with pulses of $9.4 \mathrm{keV}(1.32 \AA$ wavelength) X-rays, $40 \mathrm{fs}$ in duration, to a resolution of $1.9 \AA$ (58), clearly indicated that X-ray FEL pulses could be utilised effectively for structure determination from small crystals at the functional temperature of their constituent molecules. Cryogenic cooling of samples was clearly not required, since the sample is vaporised in any case. This also meant each crystal is single use, providing a single snapshot diffraction pattern and the potential for time-resolved measurements of samples undergoing irreversible reactions, whether initiated by a light pulse or by bringing reactants into contact (with two streams joining in a liquid jet, for example (59)).

The serial approach was a drastic departure from the practises of macromolecular crystallography, in which crystals are usually rotated as diffraction data is being collected. The new approach dispensed with the goniometer completely, following Hajdu's scheme of measuring diffraction snapshots from a stream of particles in random and unknown orientations, but now with the great advantage that those particles were not exposed directly to the vacuum environment. A new diffraction analysis strategy was required, but it was realised that crystals gave advantages over the continuous diffraction of single molecules that the FLASH experiments had prepared for. Crystals diffract into Bragg peaks on a regular lattice, and the orientation of each crystal could be read directly from each pattern by identifying a number of those peaks using crystallographic auto-indexing programs (60). The loss of the ability to rotate crystal reflections through their diffracting condition that would have provided a full integration of intensities for accurate readings of the structure factors, was a potential serious defect. Counter to this was that powder diffraction gives fully formed 
Debye-Scherrer rings (even for a non-rotating sample) consisting of partial Bragg reflections of the many crystallites that happen to be oriented close to the diffracting condition. Each Debye-Scherrer ring has contributions from a different subset of crystals, and each crystal contributes to a different set of reflections. Nevertheless, integrating over the full arc length and width of each ring gives a set of structure factors of the molecular constituent on a common scale, since the statistical variations of one subset of crystals are close to those of every other. Serial crystallography can be thought of powder diffraction collected one crystallite at a time, with the benefit of no overlapping Bragg peaks (as can occur with Debye Scherrer rings). The approach was referred to as a Monte Carlo integration of the full Bragg reflections (60), although observed peak profiles and estimations of partiality were quickly adopted into a software suite called CrystFEL (61) to achieve higher accuracy than that simple integration method. CrystFEL is widely used and actively developed today (62) and has been joined by other software projects $(63,64)$.

Some of the first applications of serial crystallography obtained structures from small crystals, such as those of cathepsin B (65) grown in living cells, natural mosquito larvacide crystals found in spores (66), as well as another pesticide called granulovirus that forms a crystalline shell around the viral body (33). In other cases, structures of radiation-sensitive metalloproteins such as photosystem II $(67,68,69)$, photosynthetic reaction centre $(70)$, and copper-containing nitrite reductase (71) could be determined at room temperature and without confounding effects due to photo-reduction of active centers. While liquid jets provide a low scattering background and the ideal medium for carrying crystals to the X-ray beam, they were often problematic, especially when the medium required salts or high concentrations of polyethylene glycol. Improved nozzle designs that form a jet using a sheath liquid that encloses and focuses the sample-containing medium has substantially reduced jet failures. Such a coaxial jet formed by an electric field was used to obtain a roomtemperature structure of a ribosomal subunit using only $360 \mu \mathrm{L}$ of sample solution (72) and a gas-focused coaxial jet flowing at $5 \mu \mathrm{L} \mathrm{min}{ }^{-1}$ was used for a room-temperature structure of RNA polymerase II from $10.8 \mathrm{mg}$ of sample (73). Membrane proteins such as the extremely important family of G-protein-coupled receptors (GPCRs) were known to form micro-crystals in lipidic cubic phase (LCP), most too small for conventional analysis. Uwe Weierstall at ASU developed a nozzle that extrudes the highly viscous LCP material across the beam, which has been used to solve many GPCR structures (74). A recent review of serial femtosecond crystallography of GPCRs is given by Stauch and Cherezov (75). Unlike liquid jets which run at speeds between 10 and $100 \mathrm{~m} \mathrm{~s}^{-1}$, the viscous LCP can be extruded at a speeds of several millimeters per second that are well matched to the $120 \mathrm{~Hz}$ arrival rate of X-ray pulses. This more efficient usage results in a consumption of $100 \mu \mathrm{g}$ to $1 \mathrm{mg}$ for a structure (75).

The ultimate sample efficiency may be achieved by applying crystals to a support membrane which is scanned through the beam $(76,77)$. This approach is favoured for crystals too large for liquid jets, and even for those large enough to be exposed by a focused beam at several positions $(78,79)$. Patterned chips place crystals in defined positions which can be interrogated with X-ray FEL pulses at the $120 \mathrm{~Hz}$ of the LCLS, making good use of pulses and available crystals. Realising the benefits of FEL pulses for crystallography with larger crystals, and now having a range of options for sample delivery and data collection, the MFX beamline was built at the LCLS in 2016 to meet growing demands for these and goniometer-based experiments $(80,81)$.

Inefficient consumption due to too fast jet flow is not the problem for the latest genera- 
tion of X-ray FELs, such as the European XFEL which will deliver up to 27000 pulses per second. This facility actually produces ten bursts of pulses per second, with pulses within the burst arriving as little as $220 \mathrm{~ns}$ after each other. In such a case a liquid jet speeding at $100 \mathrm{~m} \mathrm{~s}^{-1}$ would be as efficient as an LCP extrusion moving at about $2.5 \mathrm{~mm} \mathrm{~s}^{-1}$, at least during the period of the X-ray burst. First tests have shown diffraction can indeed be collected at megahertz rates using nozzles designed to generate the necessary high speeds (82), and new detectors can match this rate for short bursts (83). However, the jets would need to be pulsed ten times per second, coinciding with the X-ray bursts, in order not to waste more than $99 \%$ of the sample during the X-ray dark periods. If sample was flowing not more than say 10 times longer than the X-ray bursts, it may be possible to acquire 10000 diffraction patterns from about $3 \times 10^{5}$ pulses (a $3 \%$ "hit" fraction) in less than $15 \mathrm{~s}$ from less than $0.1 \mu \mathrm{L}$ of material. A potential method would pulse the sample into the continuously flowing jet of the double-flow focused nozzle (73).

Opportunities for time-resolved serial crystallography began to be explored once it was clear that small changes of structure factors could be measured by serial crystallography $(84,85)$. The femtosecond pulses could resolve much faster processes occurring in photoactivated enzymes than possible before, to reach times below 1 ps to observe the first atomic motions in a reaction. Although the synchronisation between the the arrival of the optical laser pulse (to trigger the photoreaction) and the X-ray pulse (to measure the diffraction) fluctuated by $100 \mathrm{fs}$ or more, methods were developed to precisely measure the delay on each pulse (86) to piece together a time series from the inherent variation. In this way the the isomerisation of the chromophore in photoactive yellow protein was observed to occur within $590 \mathrm{fs}(87)$ and dynamics at a similar timescale were observed in rhodopsin prior to isomerisation (88). Due to the strong absorbance of photoactive proteins, light only penetrates several micrometers into these materials. The small crystals thus gave another advantage that the photoreaction occurs throughout the complete volume probed by X-rays, ensuring much larger structure factor differences between the dark and the optically "pumped" crystals than achieved in prior synchrotron measurements. Over longer timescales, intermediate structures of photosystem II undergoing the water splitting reaction were captured, and efforts are continuing on this important system within three different collaborative efforts $(89,69,68)$.

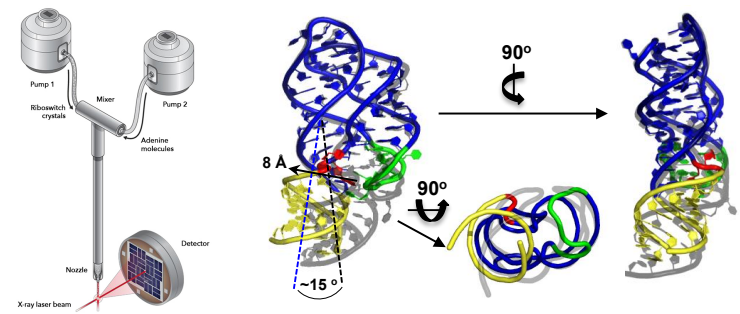

Figure 2

Using mix-and-inject serial crystallography (schematic on left), intermediate structures of a mRNA aptamer were obtained at as a ligand diffused into the crystals. At right are the apo1 (coloured) and ligand-bound (grey) structures superimposed. After mixing the crystals changed form from $P 2_{1}$ symmetry to $P 2_{1} 2_{1} 2_{1}$ (90)

Jetting the crystals across the X-ray beam in a liquid medium led to schemes of 
continuous-flow mixing to measure conformational changes and reactions upon ligand binding and their kinetics on the scale of milliseconds to many seconds. Diffusion times of a substrate into a crystal - proportional to crystal surface area — are as low as $100 \mu \mathrm{s}$ for $1 \mu \mathrm{m}$ wide crystals (91). One of the first demonstrations of this "mix-and-inject" technique (91), illustrated in Figure 2, measured conformers of RNA riboswitches, aptamers of messenger RNA that bind to a particular ligand to regulate gene expression (90). The magnitude of the structural changes on binding were significant, especially when compared with the unit cell of the crystal. Yet the microcrystals did not constrain these movements and seemingly could adapt to the huge strains that resulted, even undergoing a change in symmetry. Such effects had frustrated efforts dating back to the earliest attempts to study structural transformations in crystals of hemoglobin which would crack and become disordered on exposure to air (92). This was still a limiting factor for time-resolved Laue diffraction measurements using synchrotron radiation, which needed millimetre-sized crystals that were cooled to slow down reactions (93). With the small crystals now accessible, below $1 \mathrm{\mu m}^{3}$ in volume, most of the molecules lie close to the surface and strain can be more easily relieved, finally making it possible to capture the short-lived intermediate states of enzyme-catalyzed or ligand-binding reactions.

By removing the restriction to obtain as much data as possible from one or few crystals as is common in conventional crystallography, the tools and methodology of serial crystallography provided the opportunity to reduce the dose almost arbitrarily when crystal volumes of many cubic micrometers are available. This strategy was first demonstrated using synchrotron radiation on cryogenically-cooled in vivo grown crystals of cathepsin B which were initially thought too small for such a facility (94). The dose was reduced even further for room-temperature measurements (95) at exposure times of $3 \mathrm{~ms}$, opening up the mix-and-inject style of the measurement of structural kinetics (96). Many synchrotron facilities are introducing beamline instrumentation and, crucially, new detectors for serial crystallography (97). Undulator radiation at these facilities has a relative bandwidth of about $2 \%$, giving a more complete integration of Bragg intensities in a single snapshot pattern than with a monochromatic beam $(98,99)$, reducing the number of observations required. Combined with the higher flux, this "pink-beam" serial crystallography allows exposures down to $100 \mathrm{ps}$ of single pulses from crystals about $10 \mu \mathrm{m}$ wide (100).

\section{FROM CRYSTALS TO SINGLE MOLECULES}

\subsection{Diffraction signal versus exposure}

Many of the published accounts of serial diffraction experiments (mostly crystallography), from both X-ray FELs and synchrotron facilities, are summarised in a log-log plot of object size versus dose shown in Figure 3. The exposure to each particle can be expressed in terms of the incident X-ray fluence (number of photons per unit area), $I_{0}$. The product of fluence with the mean cross section of the atoms in the sample $\bar{\sigma}$ gives the number of scattered photons per atom for a particular material, shown as an alternative scale of the abscissa $\left(\bar{\sigma}=7 \mathrm{~b}=7 \times 10^{-16} \mathrm{\mu m}^{2}\right.$, for a typical protein at $8 \mathrm{keV}$ ). Dose, proportional to the number of photons absorbed per atom, is also proportional to this scale. The volume of the crystals or particles, approximated as the cube of the object width given on the ordinate, is proportional to the total number of atoms contained in the object, equal to the product $N_{\text {atom }} N_{\text {asym }}$ of the number of atoms per asymmetric unit and the number of asymmetric units in the object. Single particles have $N_{\text {asym }}=1$. Multiplying the number 
of atoms in the sample by the scattered photons per atom, $I_{0} \bar{\sigma} N_{\text {atom }} N_{\text {asym }}$, gives the estimated total photon count in the diffraction pattern, forming straight diagonal lines on the plot. Most experiments are carried out with objects that are at least big enough to give more than 10000 scattered counts, and the spread of the data points indicates that the diffraction signal strength per shot is a key parameter in such experiments. Interestingly, the experiment that obtained a structure with the lowest counts per pattern was actually conducted with a laboratory X-ray tube (101), shown at the top left of the plot. 8.8 million diffraction patterns were collected from one large lysozyme crystal with an average of only 200 photons recorded per pattern. The dataset was assembled without knowledge of the crystal orientations to a resolution of $1.5 \AA$. If it had been carried out on 8.8 million individual crystals, the dose would have been less than $1 \mathrm{mGy}$.

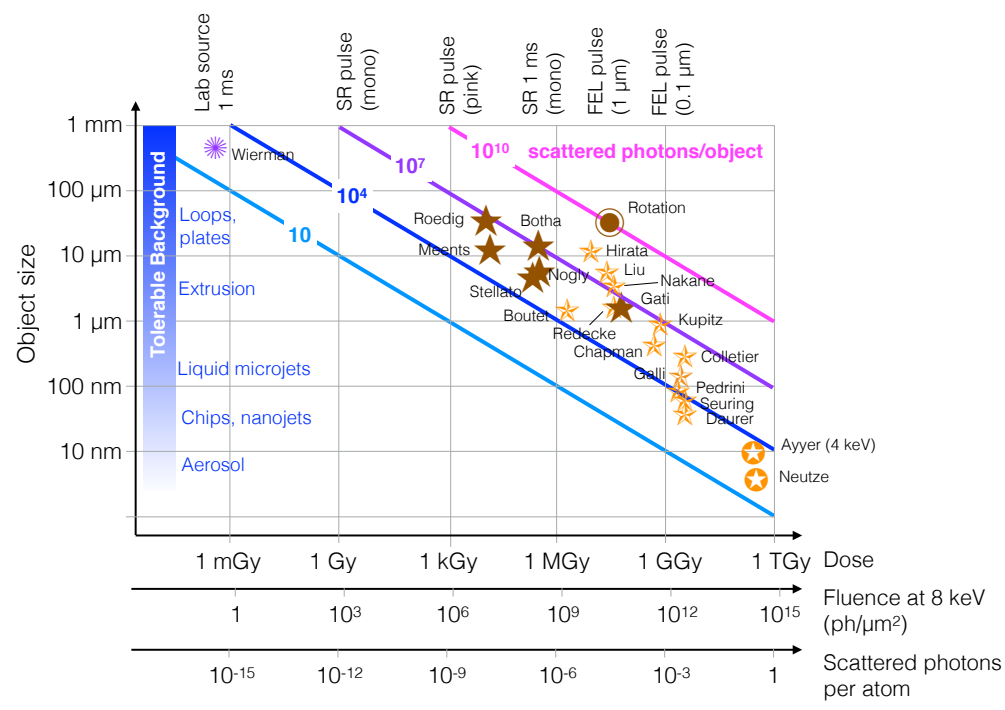

\section{Figure 3}

A selection of serial crystallography experiments plotted on a log-log graph of crystal size versus dose (or equivalently X-ray fluence or scattered photons per atom). The crystal size is computed as the cube root of the illuminated volume. The total diffracted signal for a particular stoichiometry is proportional to the product of the number of diffracted photons per atom and object volume, shown as solid lines. Orange symbols correspond to XFEL experiments, brown to experiments at synchrotron radiation facilities, and purple illustrates an experiment using a laboratory source. References are as follows: Wierman (101), Roedig (102), Meents (100), Stellato (95), Nogly (103), Botha (104), Boutet (58), Hirata (78), Liu (105), Nakane (106), Redecke (65), Gati (94), Chapman (49), Kupitz (68), Colletier (66), Pedrini (107), Galli (33), Seuring (108), Daurer (109).

The laboratory-source experiment was not primarily motivated by the attractions of low-dose crystallography, but to demonstrate the ability to interpret patterns with low signals. With only 200 counts, every pattern looked entirely random and certainly no Bragg peaks could be discerned or indexed. In the absense of any information about the orientation of particles, there need be enough information encoded in each diffraction pattern to determine that orientation with respect to all others, equivalent to determining whether two noisy patterns represent the same view of the object or two different views (110). The work of Wiermann et al. (101) showed that the 200 photon counts indeed provided enough 
information to assemble data into a three-dimensional array. This was achieved with the Expansion-Maximization-Compression (EMC) algorithm developed by Loh and Elser (111) that was originally applied to the iron-oxide nanoparticle diffraction at FLASH (43). Similar to methods in cryo-electron microscopy (112), this algorithm iteratively enforces consistency of all individual patterns with a single $3 \mathrm{D}$ reciprocal-space array of diffraction intensities by updating the values in that array by placing the measured patterns at particular orientations, weighted by probabilities given by a statistical test.

At the other corner of the plot, where estimated signals are shown for single molecules of lysozyme (18) and RNA polymerase II (113) at a dose of more than 100 GGy almost fifteen orders of magnitude larger than the X-ray tube experiment, one must consider background scattering from the beamline or the medium carrying the sample. The measurement of a strongly-diffracting crystal with a weak beam can be made without extraneous counts, but a weakly-diffracting molecule illuminated by a strong beam will compete with any other weakly-scattering structures in the beam. An illustrative example that allows quantitative examination is an experiment carried out on "invisibly small" crystals of a protein called polyhedrin that encapsulate granuloviruses (33). These occlusion bodies have a small variation of size, with crystalline volumes of only $0.0096 \mathrm{\mu m}^{3}$, corresponding to 9000 unit cells, and with 24 asymmetric units per unit cell: $N_{\text {asym }}=216000$. X-ray pulses focused to $10^{12}$ photons $\mu \mathrm{m}^{-2}$ (about $1.3 \mathrm{GGy}$ dose) gave average counts per diffraction pattern (in Bragg peaks) of about 100000 , corresponding to one photon count for every 3000 atoms in the crystal. The diffuse background, originating primarily from scattering from atoms in the $3 \mu \mathrm{m}$ diameter jet, contained about 20 times as many counts than the signal. It appears feasible with the obtained background to reduce crystal size still further to volumes of about 100 unit cells, giving only 1000 photons per pattern. Indeed, this was the case for the measurement of two-dimensional crystals of a mutant of bacteriorhodopsin at $4 \AA$ resolution, where a low enough background was achieved by mounting the crystals in a glucose layer on a silicon nitride membrane (114), and $2.9 \AA$ resolution diffraction from $30 \mathrm{~nm}$ wide crystalline fibrils of amyloids (36). Daurer et al. (109) provide a detailed account of signal and noise levels that have been achieved in diffraction measurements of $40 \mathrm{~nm}$ diameter Omono River virus particles at the LCLS, finding signals of several thousand photons per pattern to a resolution of about $5 \mathrm{~nm}$ and a background as low as several hundred counts. The focused X-ray intensity was found to be about 20 times lower than anticipated from beamline simulations (115). Diffraction patterns of single Rice Dwarf viruses (116), carried out as part of the Single Particle Initiative achieved diffraction signal above noise to a resolution of $5.9 \AA$.

Higher pulse fluence provides a greater signal for single particle diffraction, and with focusing optics with an acceptance matched to the source, it should be possible to reach fluences exceeding $10^{14}$ photons $\mu \mathrm{m}^{-2}$ at sources such as the LCLS and European XFEL which produce millijoule pulses. At SACLA pulse fluences exceeding $5 \times 10^{12}{\text { photons } \mathrm{um}^{-2}}^{-2}$ were achieved by focusing $42 \mu \mathrm{J}$ pulses to a spot of $55 \mathrm{~nm} \times 30 \mathrm{~nm}$ with a duration of $7 \mathrm{fs}(117)$. Increased pulse energies from the source will give correspondingly higher fluences, which must preserve pulse durations to below $20 \mathrm{fs}$. Design changes to achieve the necessary terawatt powers have been proposed (118). At such fluence, a large complex, such as RNA polymerase II for example (113), containing 31000 atoms, would yield a diffraction signal per pulse, $I_{p} \approx 2000$ photons ( 0.07 photons per atom), of similar signal achieved in the highresolution $2 \mathrm{D}$ crystal and fibre experiments mentioned above. Reducing the photon energy to $4 \mathrm{keV}$ would give a factor of four times higher scattering cross section, and a factor of two 
more photons from the FEL, to reach the scattering saturation regime discussed above (23), with about 0.5 photons scattered per atom, but requiring sub-femtosecond pulses. This can be compared with cryo-electron microscopy, where a tolerable dose of about $10 \mathrm{e} / \AA$ gives rise to 0.06 electrons in an image per atom, although electron microscopy has the advantage that subsequent over-dosed images can be used to locate and orient particles (but not used for the final structure determination).

\subsection{Number of patterns}

The number of snapshot patterns of crystals or single particles needed to solve a structure depends on the SNR of the individual patterns, the symmetry of the crystal or particle, the variability of parameters on which the diffraction depends on from shot to shot (such as the chaotic spectrum of FEL pulses), and systematic errors (such as introduced by incorrect knowledge of the absolution positions of all detector pixels). These factors influence the final accuracy of the merged data, the requirements of which depend on the method of phasing or specific use of the data (such obtaining time-dependent structures). As such, as few as 100 patterns gave structures of myoglobin (119) using molecular refinement, compared with about 60000 indexed patterns used for the first demonstration of a de novo protein crystal structure - determination by single-wavelength anomalous diffraction (SAD) of lysozyme crystals soaked with a gadolinium compound (120). Over twice that many were required for the even more challenging task of sulfur-SAD phasing of lysozyme (106) and thaumatin (121). Experiences with different detector systems (which necessarily all employ cutting-edge technologies $(122,44,123,83))$ suggest that their noise and gain characteristics impact data quality and that significant improvements can be expected.

In the absense of all noise other than photon counting statistics, the total aggregated counts required to reconstruct electron density maps at near-atomic resolution, as determined by simulation for single particles, is about $10^{3}$ per atom in the asymmetric unit ${ }^{3}$. In this ideal case, the signal can be partitioned between many snapshots, as long as there is a minimum signal to ensure orientation assignment of each pattern and there are enough patterns to sample the $3 \mathrm{D}$ volume of reciprocal space to full completeness (dependent on the symmetry of the particle). Given a signal per pattern of $I_{p}=I_{0} \bar{\sigma} N_{\text {atom }} N_{\text {asym }}$ and a total required signal of $T=10^{3} N_{\text {atom }}$, we find that the number of patterns is independent of molecule size, at $T / I_{p}=10^{3} /\left(\bar{\sigma} I_{0} N_{\text {asym }}\right)$. For expected fluences of $10^{14}$ photons $_{\mu m^{-2}}$, the number of patterns is about 15000 for single particles $\left(N_{\text {asym }}=1\right)$, but this number increases rapidly with the inverse square of the achievable SNR of individual patterns. The refinement of the polyhedrin structure of granuloviruses to a resolution of $2.9 \AA$ was achieved with 82000 patterns. Given the pulse fluence of $I_{0}=10^{12}$ photons $\mu^{-2}$, the number of asymmetric units per crystal of $N_{\text {asym }}=216000$, and an estimated SNR at the highest resolution of 0.02 , we would expect $\approx 16000$ patterns would be needed, comparable to number used. The larger micro-crystals used for most serial crystallography measurements should yield far more than the required $10^{3} N_{\text {atom }}$ photons in a single shot. Thus, the number of patterns required to solve a structure in these experiments is dictated almost entirely by noise due to background scattering and the need to average over fluctuations of pulse parameters and crystal shapes, sizes, and quality. Methodologies for single-particle experiments could therefore still provide substantial gains for crystallography (125).

\footnotetext{
${ }^{3}$ e.g. Ref. (124) gives simulations for $4 \times 10^{3}$ scattered photons per atom
} 


\subsection{Single-molecule diffraction}

After continued development since first experiments at LCLS in 2009 and SACLA in 2012, consisting of perhaps 1500 hours in total beamtime made available at these two operating hard X-ray FEL facilities, much progress has been made on addressing the goal of singlemolecule diffraction as proposed almost 20 years ago. As seen here the challenges are: a method to introduce molecules to the beam while generating no more than 100 background scattering photon counts in diffraction pattern; achieving a stream of reproducible and uncontaminated particles; acquiring enough patterns at high enough X-ray intensity to provide a dataset of tens of thousand to millions of patterns; and the interpretation of the noisy diffraction data. These challenges are interdependent, requiring the continued engagement of the community and facilities to solve. Some datasets of single particle diffraction from virus particles have been made available $(116,126)$.

Acquiring diffraction patterns of non-crystalline objects at low enough signal to noise ratio (SNR) is inextricably linked with the methodology to introduce molecules to the beam. Two promising methods are focused aerosol particle injectors of the kind that have been used since the first FLASH experiments $(41,53,127)$, and thin solid supports, such as graphene, that can be scanned through the beam $(128,129,108)$. Vacuum environments are used to avoid air scatter: $1 \mathrm{~mm}$ thickness of air or $20 \mathrm{~mm}$ helium creates as much diffuse scattering as a $1 \mu \mathrm{m}$ liquid jet (130). A challenge for aerosolisation are contaminants, which concentrate in evaporating drops. In principle, the averaging of diffraction data of single molecules that are surrounded with varying numbers of bound water molecules or even embedded in droplets of different sizes should yield the structure of the reproducible object with the variable additions appearing as an unstructured solvent. However, the 3D data assembly algorithms must contend with such variation - a much bigger challenge. Aerosol injection has not yet reached the required efficiencies to yield a number of patterns approaching $15000 / \mathrm{SNR}^{2}$, but higher pulse repetition rates, such as $27000 \mathrm{~s}^{-1}$ at the European XFEL, may enable this.

Given that the signals per atom could be comparable to or higher than those achieved in cryo-EM, it would appear that XFEL diffraction could tolerate as much noise generated from the medium as does that method. That is, a liquid thickness of 100 to $200 \mathrm{~nm}$ might be tolerable, especially for resolutions worse than about $4 \AA$, considering that in Fourier space most of the diffuse scattering from liquid water occurs at scattering angles corresponding resolutions higher than that. Liquid jets (131) and sheets (132) approaching this thickness have been achieved. However, unlike microscopy, scattering from extraneous atoms occurs throughout the interaction volume of the beam with the medium. The X-ray beam should therefore be focused to a spot size about as large as the particle under study. In this case, the beam shape and offset relative to the particle must be accounted for in the data assembly and structure recovery steps. The tolerance for low SNR may be increased if the orientation of the particles are constrained. Non-spherical objects align their major axes parallel to the flow of a microjet (133), similar to one-dimensional alignment of polarisable molecules using optical laser fields $(134,45,135)$. The ratio of the volume of the object of interest to background-producing solvent can be increased by increasing particle density, to illuminate several objects per snapshot pattern. The short snapshot exposures of FEL pulses, shorter than rotation times of molecules, gives coherent diffraction patterns that can be interpreted through a correlation analysis to gain the $3 \mathrm{D}$ structure-factor dataset of the single particle $(136,137,138,139)$.

Many approaches have been proposed and tested to increase the continuous diffraction 
signals of single objects by suitable engineering of the sample. Attaching a known and strongly scattering object to the object $(140)$ or placing one nearby $(141,142)$ can boost the interpretable signal in the diffraction pattern far above noise that would have overwhelmed diffraction from the weak object alone. Such reference objects aid in phasing, and can be introduced as a second stream of aerosolised particles that intersects with the objects of interest as they pass through the X-ray beam (143). If the objects can be fully aligned in all three dimensions then signal can be aggregated over many pulses and particles, without requirement on the SNR of individual patterns, with the resolution determined by the degree of alignment (46). Conceptually, this is akin to spreading the signal over the billions of molecules in a crystal, but by avoiding the periodicity of the crystal the full information content of the diffraction of single objects is made accessible (see "IMAGING WITHOUT LENSES"). As it turns out, a crystal is actually a very good starting point to achieve an ensemble of aligned molecules. Small random translations of complexes in photosystem II crystals (of $1.4 \AA$ ) destroys conditions for constructive interference of intensities from all complexes at high scattering angles that would otherwise form Bragg peaks. Instead, an incoherent addition of the single-particle diffraction of the photosystem II complex is revealed at near-atomic resolution, which can be iteratively phased (37). Such crystals may provide a suitable platform for further developments of algorithms or preparation of host-guest systems (144) for the study of of small proteins.

The acquisition of continuous diffraction patterns of single molecules would provide complete and unbiased electron density maps of these structures, without crystallisation, measured at physiological temperatures (particularly if measured in a liquid environment). As well demonstrated in cryo-electron microscopy, measuring particles one at a time enables the discrimination of multiple conformations or binding poses that the molecules may adopt. The algorithms to achieve this - EMC and manifold embedding - are conceptually similar as applied to real-space data of electron microscopy or reciprocal-space data of diffraction $(145,112)$. Ourmazd introduced the ability to not only to sort conformations but to determine their principle components (145). These can then be used to map out the energy landscape of thermally-driven processes $(146,147)$. The more often a particular conformer is observed, the more stable it must be and, conversely, the rarest events are those furthest from equilibrium. Since the relative energies of two conformations depends on the exponent of the ratio of their populations, Spence has argued that high-throughput single-particle FEL measurements may acquire many millions of measurements, enough to observe map energy differences exceeding that of the energy available from ATP per molecule (148). Diffraction measurements of single particles in liquid environments would also open up time-resolved measurements of conformational dynamics through mixing or optical stimulation, and the ability to capture rare instances of structural transitions.

\section{FUTURE ISSUES}

1. The "cost of entry" for measurements at FELs is diminishing with the creation of more facilities and beamlines, higher efficiency and capacity (such as increased pulse repetition), the maturation and standardisation of technologies, and parallel techniques at synchrotron facilities.

2. With full automation, tens to hundreds of structures could be obtained per hour. The quickest way to screen and optimise crystals - or other material forms - would be via serial diffraction using FEL pulses. 


\section{X-RAYS FROM LINEAR ACCELERATORS}

To generate the most intense X-ray beams ever made, a beam of electrons is accelerated to close to the speed of light and shot through an "undulator" consisting of a periodic array of magnets, with poles alternating in direction to force the electrons along a slalom course. Like a radio antenna, this oscillation of charge produces radiation at the frequency of oscillation, which due to relativistic effects appears as X-rays to experimenters in the laboratory. By using a linear accelerator (instead of a ring) electron bunches can be first compressed to pulses of femtosecond duration. The high density of electrons then creates a positive feedback as the co-moving radiation drives them into sheets separated by the oscillation period, like ripples in sand. The stronger the radiation, the more structured the electron bunch becomes, producing even stronger radiation through interference. This process grows exponentially over many hundreds of undulator periods, giving coherent laser-like X-ray pulses of femtosecond duration that can be focused to intensities (number of photons per unit area and time) more than a billion times higher than produced at a synchrotron facility.

\section{IMAGING WITHOUT LENSES}

When illuminated with a coherent X-ray beam, waves scattered from individual atoms in an object will interfere at a detector placed far away, producing a diffraction pattern. A striking example is crystal diffraction, where the regularity causes constructive addition of waves from each repeating unit in the object, at discrete "Bragg peaks". A single non-repeating object, in contrast, gives rise to a smoothlyvarying pattern. In both cases the pattern can be interpreted as the Fourier transform of the object's electron density. The detector records only the strength of the diffracted wave but not its phase. If the diffraction phases were known, an inverse Fourier transform would produce a map of that electron density, in the same way as a perfect lens recombines the scattered light into an image. The information content of the measured intensities exceeds that to describe the single object, meaning that phases can be recovered from the measurement alone using iterative algorithms (149). An exception is the crystal: Bragg peaks miss up to half the information in each dimension, making crystal structure determination much more difficult than for single particles.

3. Measurements of the intermediate states and evolution of structures based on mixing and light triggering will be combined with investigations of other external influences such temperature, electric field, or chemical environment, to gain greater insights into protein function.

4. Single-molecule imaging requires improvements in sample delivery to achieve low background and increased efficiency. Ensemble measurements of single particles will map out conformational landscapes and reaction pathways.

5. Sample engineering could lead to greater availability of information for structure determination, avoiding model biases and providing structures from difficult samples. Examples include induced translational disorder of crystals, strongly-scattering ref- 
erence structures, particle alignment, finite crystals, and host-guest systems.

\section{DISCLOSURE STATEMENT}

The author is not aware of any affiliations, memberships, funding, or financial holdings that might be perceived as affecting the objectivity of this review.

\section{ACKNOWLEDGMENTS}

The author acknowledges the FEL facilities, FLASH, LCLS, FERMI, and SACLA, that have welcomed and supported the work reviewed here and the large number of collaborators and community who contributed to methods of structure determination at those facilities. Also acknowledged is the funding of the Helmholtz Association and DESY to the author and his group at the Center for Free-Electron Laser Science.

\section{LITERATURE CITED}

1. Garman EF. 2014. Developments in X-ray crystallographic structure determination of biological macromolecules. Science 343:1102-1108

2. Berman H, Westbrook J, Feng Z, Gilliland G, Bhat T, et al. 2000. The protein data bank. Nucl. Acids Res. 28:235-242

3. Owen RL, Rudino-Pinera E, Garman EF. 2006. Experimental determination of the radiation dose limit for cryocooled protein crystals. Proc. Nat. Acad. Sci. 103:4912-4917

4. Garman EF, Schneider TR. 1997. Macromolecular Cryocrystallography. J. Appl. Cryst. 30:211237

5. Dubochet J. 2012. Cryo-EM - the first thirty years. J. Microsc. 245:221-224

6. Elmlund D, Elmlund H. 2015. Cryogenic electron microscopy and single-particle analysis. Ann. Rev. Biochem. 84:499-517

7. Kühlbrandt W. 2014. Cryo-EM enters a new era. eLife 3:03678

8. Seddon EA, Clarke JA, Dunning DJ, Masciovecchio C, Milne CJ, et al. 2017. Short-wavelength free-electron laser sources and science: a review. Rep. Prog. Phys. 80:115901

9. Wood L, Chapline G. 1974. Towards gamma-ray lasers. Nature 252:447-450

10. Chapline G, Wood L. 1975. X-ray lasers. Phys. Today 28:40-48

11. Breedlove JR, Trammell GT. 1970. Molecular microscopy: Fundamental limitations. Science 170:1310-1313

12. Solem JC, Baldwin GC. 1982. Microholography of living organisms. Science 218:229-235

13. Solem JC, Chapline GF. 1984. X-ray biomicroholography. Opt. Eng. 23:193-203

14. Solem JC. 1986. Imaging biological specimens with high-intensity soft x rays. J. Opt. Soc. Am. B 3:1551-1565

15. London RA, Rosen MD, Trebes JE. 1989. Wavelength choice for soft x-ray laser holography of biological samples. Appl. Opt. 28:3397-3404

16. Doniach S. 1996. Studies of the Structure of Matter with Photons from an X-ray Free-Electron Laser. J. Synchr. Rad. 3:260-267

17. Wilmanns M. 2000. Future structural biology applications with a free-electron laser - more than wild dreams? J. Synchr. Rad. 7:41-46

18. Neutze R, Wouts R, van der Spoel D, Weckert E, Hajdu J. 2000. Potential for biomolecular imaging with femtosecond x-ray pulses. Nature 406:753-757 
19. Emma P, Akre R, Arthur J, Bionta R, Bostedt C, et al. 2010. First lasing and operation of an ångstrom-wavelength free-electron laser. Nat. Photon. 4:641-647

20. Yabashi M, Tanaka H, Ishikawa T. 2015. Overview of the SACLA facility. J. Synchr. Rad. 22:477-484

21. Altarelli M, Brinkmann R, Chergui M, Decking W, Dobson B, et al. 2007. The European X-Ray Free-Electron Laser technical design report. Tech. rep., DESY XFEL Project Team

22. Classen A, Ayyer K, Chapman HN, Röhlsberger R, von Zanthier J. 2017. Incoherent diffractive imaging via intensity correlations of hard x rays. Phys. Rev. Lett. 119:053401

23. Son SK, Young L, Santra R. 2011. Impact of hollow-atom formation on coherent x-ray scattering at high intensity. Phys. Rev. A 83:033402

24. Ziaja B, van der Spoel D, Szöke A, Hajdu J. 2001. Auger-electron cascades in diamond and amorphous carbon. Phys. Rev. B 64:214104

25. Caleman C, Ortiz C, Marklund E, Bultmark F, Gabrysch M, et al. 2009. Radiation damage in biological material: Electronic properties and electron impact ionization in urea. EPL (Europhys. Lett.) 88:29901

26. Chapman HN, Caleman C, Timneanu N. 2014. Diffraction before destruction. Phil. Trans. R. Soc. Lond. B 369:20130313

27. Nass K, Foucar L, Barends TRM, Hartmann E, Botha S, et al. 2015. Indications of radiation damage in ferredoxin microcrystals using high-intensity X-FEL beams. J. Synchr. Rad. 22:225238

28. Rudenko A, Inhester L, Hanasaki K, Li X, Robatjazi SJ, et al. 2017. Femtosecond response of polyatomic molecules to ultra-intense hard x-rays. Nature 546:129-132

29. Skinner CH, DiCicco DS, Kim D, Rosser RJ, Suckewer S, et al. 1990. Contact microscopy with a soft X-ray laser. J. Microsc. 159:51-60

30. Da Silva L, Trebes J, Balhorn R, Mrowka S, Anderson E, et al. 1992. X-ray laser microscopy of rat sperm nuclei. Science 258:269

31. Ackermann W, Asova G, Ayvazyan V, Azima A, Baboi N, et al. 2007. Operation of a freeelectron laser from the extreme ultraviolet to the water window. Nat. Photon. 1:336-342

32. Matthews D, Rosen M. 1988. Soft-x-ray lasers. Sci. Am. 259:86-91

33. Gati C, Oberthuer D, Yefanov O, Bunker RD, Stellato F, et al. 2017. Atomic structure of granulin determined from native nanocrystalline granulovirus using an x-ray free-electron laser. Proc. Nat. Acad. Sci. 114:2247-2252

34. Ekeberg T, Svenda M, Abergel C, Maia FRNC, Seltzer V, et al. 2015. Three-dimensional reconstruction of the giant mimivirus particle with an x-ray free-electron laser. Phys. Rev. Lett. 114:098102

35. Ekeberg T, Svenda M, Seibert MM, Abergel C, Maia FRNC, et al. 2016. Single-shot diffraction data from the mimivirus particle using an x-ray free-electron laser. Sci. Data 3:160060

36. Wojtas DH, Ayyer K, Liang M, Mossou E, Romoli F, et al. 2017. Analysis of XFEL serial diffraction data from individual crystalline fibrils. IUCrJ 4:795-811

37. Ayyer K, Yefanov OM, Oberthür D, Roy-Chowdhury S, Galli L, et al. 2016. Macromolecular diffractive imaging using imperfect crystals. Nature 530:202-206

38. Chapman HN, Barty A, Bogan MJ, Boutet S, Frank M, et al. 2006. Femtosecond diffractive imaging with a soft-x-ray free-electron laser. Nat. Phys. 2:839-843

39. Bajt S, Chapman HN, Spiller EA, Alameda JB, Woods BW, et al. 2008. Camera for coherent diffractive imaging and holography with a soft-x-ray free-electron laser. Appl. Opt. 47:1673-1683

40. Chapman HN, Hau-Riege SP, Bogan MJ, Bajt S, Barty A, et al. 2007. Femtosecond time-delay X-ray holography. Nature 448:676-679

41. Bogan M, Benner W, Boutet S, Rohner U, Frank M, et al. 2008. Single particle x-ray diffractive imaging. Nano Lett. 8:310-316

42. Treusch R, Feldhaus J. 2010. Flash: new opportunities for (time-resolved) coherent imaging of nanostructures. New J. Phys. 12:035015 
43. Loh ND, Bogan MJ, Elser V, Barty A, Boutet S, et al. 2010. Cryptotomography: Reconstructing 3D Fourier intensities from randomly oriented single-shot diffraction patterns. Phys. Rev. Lett. 104:225501

44. Strüder L, Epp S, Rolles D, Hartmann R, Holl P, et al. 2010. Large-format, high-speed, X-ray pnCCDs combined with electron and ion imaging spectrometers in a multipurpose chamber for experiments at 4th generation light sources. Nucl. Instrum. Meth. A 614:483-496

45. Spence JCH, Doak RB. 2004. Single molecule diffraction. Phys. Rev. Lett. 92:198102

46. Spence JCH, Schmidt K, Wu JS, Hembree G, Weierstall U, et al. 2005. Diffraction and imaging from a beam of laser-aligned proteins: resolution limits. Acta Cryst. A 61:237-245

47. Ganan-Calvo AM, Gonzalez-Prieto R, Riesco-Chueca P, Herrada MA, Flores-Mosquera M. 2007. Focusing capillary jets close to the continuum limit. Nat. Phys. 3:737-742

48. DePonte DP, Weierstall U, Schmidt K, Warner J, Starodub D, et al. 2008. Gas dynamic virtual nozzle for generation of microscopic droplet streams. J. Phys. D: Appl. Phys. 41:195505 (7pp)

49. Chapman HN, Fromme P, Barty A, White TA, Kirian RA, et al. 2011. Femtosecond x-ray protein nanocrystallography. Nature 470:73-77

50. Barty A, Caleman C, Aquila A, Timneanu N, Lomb L, et al. 2012. Self-terminating diffraction gates femtosecond x-ray nanocrystallography measurements. Nat. Photon. 6:35-40

51. Lomb L, Barends TRM, Kassemeyer S, Aquila A, Epp SW, et al. 2011. Radiation damage in protein serial femtosecond crystallography using an x-ray free-electron laser. Phys. Rev. B 84:214111

52. Hau-Riege SP, Bennion BJ. 2015. Reproducible radiation-damage processes in proteins irradiated by intense x-ray pulses. Phys. Rev. E 91:022705

53. Seibert MM, Ekeberg T, Maia FRNC, Svenda M, Andreasson J, et al. 2011. Single mimivirus particles intercepted and imaged with an x-ray laser. Nature 470:78-81

54. Aquila A, Barty A, Bostedt C, Boutet S, Carini G, et al. 2015. The linac coherent light source single particle imaging road map. Struct. Dyn. 2:041701

55. Sun Z, Fan J, Li H, Jiang H. 2018. Current status of single particle imaging with x-ray lasers. Appl. Sci. 8:132

56. Spence JCH, Chapman HN. 2014. The birth of a new field. Phil. Trans. R. Soc. Lond. B 369:20130309

57. Liang M, Williams GJ, Messerschmidt M, Seibert MM, Montanez PA, et al. 2015. The Coherent X-ray Imaging instrument at the Linac Coherent Light Source. J. Synchr. Rad. 22:514-519

58. Boutet S, Lomb L, Williams GJ, Barends TRM, Aquila A, et al. 2012. High-resolution protein structure determination by serial femtosecond crystallography. Science 337:362-364

59. Wang D, Weierstall U, Pollack L, Spence J. 2014. Double-focusing mixing jet for XFEL study of chemical kinetics. J. Synchr. Rad. 21:1364-1366

60. Kirian RA, Wang X, Weierstall U, Schmidt KE, Spence JCH, et al. 2010. Femtosecond protein nanocrystallography - data analysis methods. Opt. Express 18:5713-5723

61. White TA, Kirian RA, Martin AV, Aquila A, Nass K, et al. 2012. CrystFEL: a software suite for snapshot serial crystallography. J. Appl. Cryst. 45:335-341

62. White TA, Mariani V, Brehm W, Yefanov O, Barty A, et al. 2016. Recent developments in CrystFEL. J. Appl. Cryst. 49:680-689

63. Sauter NK, Hattne J, Grosse-Kunstleve RW, Echols N. 2013. New Python-based methods for data processing. Acta Cryst. D 69:1274-1282

64. Ginn HM, Evans G, Sauter NK, Stuart DI. 2016. On the release of cppxfel for processing X-ray free-electron laser images. J. Appl. Cryst. 49:1065-1072

65. Redecke L, Nass K, DePonte DP, White TA, Rehders D, et al. 2013. Natively inhibited Trypanosoma brucei cathepsin B structure determined by using an X-ray laser. Science 339:227-230

66. Colletier JP, Sawaya MR, Gingery M, Rodriguez JA, Cascio D, et al. 2016. De novo phasing with x-ray laser reveals mosquito larvicide BinAB structure. Nature 539:43-47

67. Kern J, Alonso-Mori R, Tran R, Hattne J, Gildea RJ, et al. 2013. Simultaneous femtosecond 
X-ray spectroscopy and diffraction of photosystem II at room temperature. Science 340:491-495

68. Kupitz C, Basu S, Grotjohann I, Fromme R, Zatsepin NA, et al. 2014. Serial time-resolved crystallography of photosystem ii using a femtosecond x-ray laser. Nature 513:261-265

69. Young ID, Ibrahim M, Chatterjee R, Gul S, Fuller FD, et al. 2016. Structure of photosystem II and substrate binding at room temperature. Nature 540:453-457

70. Johansson LC, Arnlund D, Katona G, White TA, Barty A, et al. 2013. Structure of a photosynthetic reaction centre determined by serial femtosecond crystallography. Nat. Comm. 4

71. Fukuda Y, Tse KM, Nakane T, Nakatsu T, Suzuki M, et al. 2016. Redox-coupled proton transfer mechanism in nitrite reductase revealed by femtosecond crystallography. Proc. Nat. Acad. Sci. 113:2928-2933

72. Sierra RG, Gati C, Laksmono H, Dao EH, Gul S, et al. 2015. Concentric-flow electrokinetic injector enables serial crystallography of ribosome and photosystem II. Nat. Meth. 13:59-62

73. Oberthuer D, Knoška J, Wiedorn MO, Beyerlein KR, Bushnell DA, et al. 2017. Double-flow focused liquid injector for efficient serial femtosecond crystallography. Sci. Rep. 7:44628

74. Weierstall U, James D, Wang C, White TA, Wang D, et al. 2014. Lipidic cubic phase injector facilitates membrane protein serial femtosecond crystallography. Nat. Comm. 5:3309

75. Stauch B, Cherezov V. 2018. Serial femtosecond crystallography of g protein-coupled receptors. Ann. Rev. Biophys. 47:377-397

76. Mueller C, Marx A, Epp SW, Zhong Y, Kuo A, et al. 2015. Fixed target matrix for femtosecond time-resolved and in situ serial micro-crystallography. Struct. Dyn. 2:054302

77. Roedig P, Vartiainen I, Duman R, Panneerselvam S, Stübe N, et al. 2015. A micro-patterned silicon chip as sample holder for macromolecular crystallography experiments with minimal background scattering. Sci. Rep. 5:10451

78. Hirata K, Shinzawa-Itoh K, Yano N, Takemura S, Kato K, et al. 2014. Determination of damagefree crystal structure of an x-ray-sensitive protein using an xfel. Nat. Meth. 11:734-736

79. Keedy DA, Kenner LR, Warkentin M, Woldeyes RA, Hopkins JB, et al. 2015. Mapping the conformational landscape of a dynamic enzyme by multitemperature and XFEL crystallography. eLife 4:e07574

80. Boutet S, Cohen AE, Wakatsuki S. 2016. The new macromolecular femtosecond crystallography (MFX) instrument at LCLS. Synchr. Rad. News 29:23-28

81. Cohen AE, Soltis SM, Gonz'alez A, Aguila L, Alonso-Mori R, et al. 2014. Goniometer-based femtosecond crystallography with x-ray free electron lasers. Proceedings of the National Academy of Sciences

82. Wiedorn MO, Awel S, Morgan AJ, Ayyer K, Gevorkov Y, et al. 2018. Rapid sample delivery for megahertz serial crystallography at X-ray FELs. IUCrJ 5

83. Allahgholi A, Becker J, Bianco L, Delfs A, Dinapoli R, et al. 2015. AGIPD, a high dynamic range fast detector for the European XFEL. J. Instrum. 10:C01023

84. Aquila A, Hunter MS, Doak RB, Kirian RA, Fromme P, et al. 2012. Time-resolved protein nanocrystallography using an x-ray free-electron laser. Opt. Express 20:2706-2716

85. Tenboer J, Basu S, Zatsepin N, Pande K, Milathianaki D, et al. 2014. Time-resolved serial crystallography captures high-resolution intermediates of photoactive yellow protein. Science 346:1242-1246

86. Harmand M, Coffee R, Bionta MR, Chollet M, French D, et al. 2013. Achieving few-femtosecond time-sorting at hard x-ray free-electron lasers. Nat. Photon. 7:215-218

87. Pande K, Hutchison CDM, Groenhof G, Aquila A, Robinson JS, et al. 2016. Femtosecond structural dynamics drives the trans/cis isomerization in photoactive yellow protein. Science 352:725-729

88. Nogly P, Weinert T, James D, Carbajo S, Ozerov D, et al. 2018. Retinal isomerization in bacteriorhodopsin captured by a femtosecond x-ray laser. Science

89. Suga M, Akita F, Hirata K, Ueno G, Murakami H, et al. 2015. Native structure of photosystem II at $1.95 \AA$ resolution viewed by femtosecond X-ray pulses. Nature 517:99-103 
90. Stagno JR, Liu Y, Bhandari YR, Conrad CE, Panja S, et al. 2017. Structures of riboswitch RNA reaction states by mix-and-inject XFEL serial crystallography. Nature 541:242-246

91. Schmidt M. 2013. Mix and inject: Reaction initiation by diffusion for time-resolved macromolecular crystallography. Adv. Cond. Matt. Phys. 2013:167276

92. Perutz MF, Bolton W, Diamond R, Muirhead H, Watson HC. 1964. Structure of haemoglobin : An X-ray examination of reduced horse haemoglobin. Nature 203:687-690

93. Moffat K. 1987. Time-resolved macromolecular crystallography. Annu. Rev. Biophys. Biophys. Chem. 18:309-332

94. Gati C, Bourenkov G, Klinge M, Rehders D, Stellato F, et al. 2014. Serial crystallography on in vivo grown microcrystals using synchrotron radiation. IUCrJ 1:87-94

95. Stellato F, Oberthür D, Liang M, Bean R, Gati C, et al. 2014. Room-temperature macromolecular serial crystallography using synchrotron radiation. IUCrJ 1:204-212

96. Beyerlein KR, Dierksmeyer D, Mariani V, Kuhn M, Sarrou I, et al. 2017. Mix-and-diffuse serial synchrotron crystallography. IUCrJ 4

97. Leonarski F, Redford S, Mozzanica A, Lopez-Cuenca C, Panepucci E, et al. 2018. Jungfrau detector: Accurate data for macromolecular crystallography. (in press)

98. White TA, Barty A, Stellato F, Holton JM, Kirian RA, et al. 2013. Crystallographic data processing for free-electron laser sources. Acta Cryst. D 69:1231-1240

99. Dejoie C, McCusker LB, Baerlocher C, Abela R, Patterson BD, et al. 2013. Using a nonmonochromatic microbeam for serial snapshot crystallography. J. Appl. Cryst. 46:791-794

100. Meents A, Wiedorn MO, Srajer V, Henning R, Sarrou I, et al. 2017. Pink-beam serial crystallography. Nat. Comm. 8:1281

101. Wierman JL, Lan TY, Tate MW, Philipp HT, Elser V, Gruner SM. 2016. Protein crystal structure from non-oriented, single-axis sparse X-ray data. IUCrJ 3:43-50

102. Roedig P, Duman R, Sanchez-Weatherby J, Vartiainen I, Burkhardt A, et al. 2016. Roomtemperature macromolecular crystallography using a micro-patterned silicon chip with minimal background scattering. J. Appl. Cryst. 49:968-975

103. Nogly P, James D, Wang D, White TA, Zatsepin N, et al. 2015. Lipidic cubic phase serial millisecond crystallography using synchrotron radiation. IUCrJ 2:168-176

104. Botha S, Nass K, Barends TRM, Kabsch W, Latz B, et al. 2015. Room-temperature serial crystallography at synchrotron X-ray sources using slowly flowing free-standing high-viscosity microstreams. Acta Cryst. D 71:387-397

105. Liu W, Wacker D, Gati C, Han GW, James D, et al. 2013. Serial femtosecond crystallography of G protein-coupled receptors. Science 342:1521-1524

106. Nakane T, Song C, Suzuki M, Nango E, Kobayashi J, et al. 2015. Native sulfur/chlorine SAD phasing for serial femtosecond crystallography. Acta Cryst. D 71:2519-2525

107. Pedrini B, Tsai CJ, Capitani G, Padeste C, Hunter MS, et al. 2014. $7 \AA$ A resolution in protein two-dimensional-crystal x-ray diffraction at linac coherent light source. Phil. Trans. R. Soc. Lond. B 369

108. Seuring C, Ayyer K, Filippaki E, Barthelmess M, Longchamp JN, et al. 2018. Femtosecond X-ray coherent diffraction of aligned amyloid fibrils on low background graphene. Nat. Comm. 9:1836

109. Daurer BJ, Okamoto K, Bielecki J, Maia FRNC, Mühlig K, et al. 2017. Experimental strategies for imaging bioparticles with femtosecond hard X-ray pulses. IUCrJ 4:251-262

110. Huldt G, Szoke A, Hajdu J. 2003. Diffraction imaging of single particles and biomolecules. J. Struct. Biol. 144:219-227

111. Loh NTD, Elser V. 2009. Reconstruction algorithm for single-particle diffraction imaging experiments. Phys. Rev. E 80:026705

112. Scheres SHW, Gao H, Valle M, Herman GT, Eggermont PPB, et al. 2007. Disentangling conformational states of macromolecules in 3D-EM through likelihood optimization. Nat. Meth. $4: 27-29$ 
113. Ayyer K, Geloni G, Kocharyan V, Saldin E, Serkez S, et al. 2015. Perspectives for imaging single protein molecules with the present design of the European XFEL. Struct. Dyn. 2:041702

114. Casadei CM, Tsai CJ, Barty A, Hunter MS, Zatsepin NA, et al. 2018. Resolution extension by image summing in serial femtosecond crystallography of two-dimensional membrane-protein crystals. IUCrJ 5:103-117

115. Pardini T, Aquila A, Boutet S, Cocco D, Hau-Riege SP. 2017. Numerical simulations of the hard X-ray pulse intensity distribution at the Linac Coherent Light Source. J. Synchr. Rad. 24:738-743

116. Munke A, Andreasson J, Aquila A, Awel S, Ayyer K, et al. 2016. Coherent diffraction of single rice dwarf virus particles using hard x-rays at the linac coherent light source. Sci. Data 3:160064

117. Mimura H, Yumoto H, Matsuyama S, Koyama T, Tono K, et al. 2014. Generation of $10^{20} \mathrm{~W} \mathrm{~cm}^{-2}$ hard X-ray laser pulses with two-stage reflective focusing system. Nat. Comm. $5: 3539$

118. Geloni G, Kocharyan V, Saldin E. 2010. Scheme for generation of fully-coherent, TW power level hard X-ray pulses from baseline undulators at the European X-ray FEL. arXiv 1007.2743

119. Uervirojnangkoorn M, Zeldin OB, Lyubimov AY, Hattne J, Brewster AS, et al. 2015. Enabling $\mathrm{x}$-ray free electron laser crystallography for challenging biological systems from a limited number of crystals. eLife 4:e05421

120. Barends TRM, Foucar L, Botha S, Doak RB, Shoeman RL, et al. 2014. De novo protein crystal structure determination from x-ray free-electron laser data. Nature 505:244-247

121. Nass K, Meinhart A, Barends TRM, Foucar L, Gorel A, et al. 2016. Protein structure determination by single-wavelength anomalous diffraction phasing of X-ray free-electron laser data. IUCrJ 3:180-191

122. Hart P, Boutet S, Carini G, Dubrovin M, Duda B, et al. 2012. The CSPAD megapixel x-ray camera at LCLS. In Proc. SPIE, ed. SP Moeller, M Yabashi, SP Hau-Riege, pp. 85040C-11, vol. 8504. $85040 \mathrm{C}-11 \mathrm{pp}$.

123. Kameshima T, Ono S, Kudo T, Ozaki K, Kirihara Y, et al. 2014. Development of an X-ray pixel detector with multi-port charge-coupled device for $\mathrm{x}$-ray free-electron laser experiments. Rev. Sci. Instrum. 85:033110

124. Miao J, Hodgson KO, Sayre D. 2001. An approach to three-dimensional structures of biomolecules by using single-molecule diffraction images. Proc. Nat. Acad. Sci. 98:6641-6645

125. Awel S, Kirian RA, Wiedorn MO, Beyerlein KR, Roth N, et al. 2018. Femtosecond X-ray diffraction from an aerosolized beam of protein nanocrystals. J. Appl. Cryst. 51:133-139

126. Reddy HKN, Yoon CH, Aquila A, Awel S, Ayyer K, et al. 2017. Coherent soft x-ray diffraction imaging of coliphage PR772 at the Linac coherent light source. Sci. Data 4:170079

127. Roth N, Awel S, Horke DA, Küpper J. 2018. Optimizing aerodynamic lenses for single-particle imaging. J. Aerosol Sci. 124:17 - 29

128. Wierman JL, Alden JS, Kim CU, McEuen PL, Gruner SM. 2013. Graphene as a protein crystal mounting material to reduce background scatter. Journal of Applied Crystallography 46:1501-1507

129. Hunter MS, Segelke B, Messerschmidt M, Williams GJ, Zatsepin NA, et al. 2014. Fixed-target protein serial microcrystallography with an x-ray free electron laser. Sci. Rep. 4:6026

130. Gruner SM, Lattman EE. 2015. Biostructural science inspired by next-generation x-ray sources. Ann. Rev. Biophys. 44:33-51. PMID: 25747590

131. Deponte D, Mckeown J, Weierstall U, Doak R, Spence J. 2011. Towards ETEM serial crystallography: Electron diffraction from liquid jets serial crystallography: Electron diffraction from liquid jets. Ultramicrosc. 111:824-827

132. Koralek JD, Kim JB, Bruza P, Curry CB, Chen Z, et al. 2018. Generation and characterization of ultrathin free-flowing liquid sheets. Nat. Comm. 9:1353

133. Popp D, Loh ND, Zorgati H, Ghoshdastider U, Liow LT, et al. 2017. Flow-aligned, single-shot fiber diffraction using a femtosecond x-ray free-electron laser. Cytoskeleton 74:472-481 
134. Stapelfeldt H, Seideman T. 2003. Colloquium: Aligning molecules with strong laser pulses. Rev. Mod. Phys. 75:543-557

135. Stern S, Holmegaard L, Filsinger F, Rouzee A, Rudenko A, et al. 2014. Toward atomic resolution diffractive imaging of isolated molecules with x-ray free-electron lasers. Faraday Discuss. 171:393-481

136. Kirian RA, Schmidt KE, Wang X, Doak RB, Spence JCH. 2011. Signal, noise, and resolution in correlated fluctuations from snapshot small-angle x-ray scattering. Phys. Rev. E 84:011921

137. Kurta RP, Donatelli JJ, Yoon CH, Berntsen P, Bielecki J, et al. 2017. Correlations in scattered x-ray laser pulses reveal nanoscale structural features of viruses. Phys. Rev. Lett. 119:158102

138. Mendez D, Lane TJ, Sung J, Sellberg J, Levard C, et al. 2014. Observation of correlated x-ray scattering at atomic resolution. Phil. Trans. R. Soc. Lond. B 369:20130315

139. Donatelli JJ, Sethian JA, Zwart PH. 2017. Reconstruction from limited single-particle diffraction data via simultaneous determination of state, orientation, intensity, and phase. Proc. Nat. Acad. Sci. 114:7222-7227

140. Shintake T. 2008. Possibility of single biomolecule imaging with coherent amplification of weak scattering x-ray photons. Phys. Rev. E 78:041906

141. Boutet S, Bogan MJ, Barty A, Frank M, Benner WH, et al. 2008. Ultrafast soft x-ray scattering and reference-enhanced diffractive imaging of weakly scattering nanoparticles. J. Electron Spectrosc. Rel. Phenom. 166-167:65-73

142. Lan TY, Li PN, Lee TK. 2014. Method to enhance the resolution of x-ray coherent diffraction imaging for non-crystalline bio-samples. New J. Phys. 16:033016

143. Gorkhover T, Ulmer A, Ferguson K, Bucher M, Maia FRNC, et al. 2018. Femtosecond x-ray fourier holography imaging of free-flying nanoparticles. Nat. Photon. 12:150-153

144. Hoshino M, Khutia A, Xing H, Inokuma Y, Fujita M. 2016. The crystalline sponge method updated. IUCrJ 3:139-151

145. Dashti A, Schwander P, Langlois R, Fung R, Li W, et al. 2014. Trajectories of the ribosome as a brownian nanomachine. Proc. Nat. Acad. Sci. 111:17492-17497

146. Frank J, Ourmazd A. 2016. Continuous changes in structure mapped by manifold embedding of single-particle data in cryo-EM. Methods 100:61 - 67

147. Haselbach D, Komarov I, Agafonov DE, Hartmuth K, Graf B, et al. 2018. Structure and conformational dynamics of the human spliceosomal bact ${ }^{\text {act }}$ complex. Cell 172:454-464.e11

148. Spence JCH. 2017. XFELs for structure and dynamics in biology. IUCrJ 4:322-339

149. Thibault P, Elser V. 2010. X-ray diffraction microscopy. Ann. Rev. Cond. Matt. Phys. 1:237255 